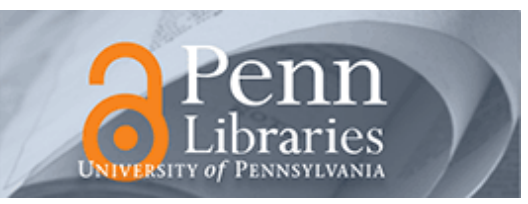

University of Pennsylvania

ScholarlyCommons

$4-5-2018$

\title{
Socio-Demographic and Clinical Determinants of Self-Care in Adults with Type 2 Diabetes: A Multicentre Observational Study
}

Davide Ausili

Emanuela Rossi

Paola Rebora

Michela Luciani

Luca Tonoli

See next page for additional authors

Follow this and additional works at: https://repository.upenn.edu/nrs

Part of the Behavioral Medicine Commons, Endocrinology, Diabetes, and Metabolism Commons, Medical Humanities Commons, Nursing Commons, and the Preventive Medicine Commons

\section{Recommended Citation}

Ausili, D., Rossi, E., Rebora, P., Luciani, M., Tonoli, L., Ballerini, E., Androni, S., Vellone, E., Riegel, B., \& Di Mauro, S. (2018). Socio-Demographic and Clinical Determinants of Self-Care in Adults with Type 2 Diabetes: A Multicentre Observational Study. Acta Diabetologica, 55 (7), 691-702. http://dx.doi.org/ 10.1007/s00592-018-1135-x

This paper is posted at ScholarlyCommons. https://repository.upenn.edu/nrs/175

For more information, please contact repository@pobox.upenn.edu. 


\title{
Socio-Demographic and Clinical Determinants of Self-Care in Adults with Type 2 Diabetes: A Multicentre Observational Study
}

\begin{abstract}
Aims

To describe self-care as defined by the Middle Range Theory of Self-Care of Chronic Illness and to identify clinical and socio-demographic determinants in a T2DM population.

Methods

A multicentre observational cross-sectional study was conducted involving 540 adults with a confirmed diagnosis of T2DM from six outpatient diabetes services in Italy. Socio-demographic and clinical data were collected from medical records. The Self-Care of Diabetes Inventory (SCODI) was used to measure self-care maintenance, monitoring, management, and confidence dimensions. For each separate scale, scores were standardized $0-100$ with higher SCODI scores indicating better self-care; a score $\geq 70$ is adequate. Multiple quantile regression models were performed to identify determinants of each self-care dimension.
\end{abstract}

Results

Self-care maintenance (median $=81.3$ ) and self-care confidence (median $=79.5$ ) were adequate in most of the subjects. Self-care monitoring was adequate in only half of the sample (median $=70.6$ ). Self-care management was poor (median $=59.4$ ). Lower self-care maintenance was associated with lower selfcare confidence $(p<0.001)$. Lower self-care monitoring was associated with being male $(p<0.001)$, having lower self-care confidence $(p<001)$, and having diabetes for $<10$ years $(p<0.001)$. Lower selfcare management was associated with being male $(p=0.002)$, being older $(p=0.005)$, having a low income $(p=0.030)$, being employed $(p=0.008)$, having missed diabetes education in the last year $(p=$ $0.002)$, and lower self-care confidence $(p<0.0001)$. Lower self-care confidence was associated with having diabetes for $<10$ years $(p=0.008)$, and having at least one comorbid condition $(p=0.006)$.

Conclusions

Determinants of self-care maintenance, monitoring, management and confidence include both clinical and socio-demographic variables. Modifiable determinants such as self-care confidence and diabetes self-care management education could be used to tailor interventions to improve diabetes self-care.

\section{Keywords}

diabetes mellitus, type 2 diabetes mellitus, self-management, chronic disease, self-efficacy, risk factors, health education

\section{Disciplines}

Behavioral Medicine | Endocrinology, Diabetes, and Metabolism | Medical Humanities | Medicine and Health Sciences | Nursing | Preventive Medicine

\section{Author(s)}

Davide Ausili, Emanuela Rossi, Paola Rebora, Michela Luciani, Luca Tonoli, Enrico Ballerini, Silvia Androni, Ercole Vellone, Barbara Riegel, and Stefania Di Mauro 


\section{TITLE}

Socio-demographic and clinical determinants of self-care in adults with type 2 diabetes: a multicentre observational study.

\section{RUNNING TITLE}

Determinants of self-care in adults with type 2 diabetes

\section{AUTHORS}

1. Davide Ausili, RN, MSc, PhD. Research Fellow in Nursing Science, Department of Medicine and Surgery, University of Milano-Bicocca, Monza, Italy.

2. Emanuela Rossi, PhD. Research Fellow in Medical Statistics, Centre of Biostatistics for Clinical Epidemiology, Department of Medicine and Surgery, University of Milan-Bicocca, Monza, Italy. 3. Paola Rebora, PhD. Assistant Professor in Medical Statistics, Centre of Biostatistics for Clinical Epidemiology, Department of Medicine and Surgery, University of Milan-Bicocca, Monza, Italy. 4. Michela Luciani, RN, MSc, PhDs, Department of Biomedicine and Prevention, University of Rome Tor Vergata, Rome, Italy. Research Fellow, Department of Medicine and Surgery, University of Milano Bicocca, Monza Italy.

5. Luca Tonoli, RN, MSc. Adjunct Professor of Medical Nursing, Undergraduate Nursing Degree, University of Brescia, Brescia Italy.

6. Enrico Ballerini, RN, MSc. Adjunct Professor of Health Management, Undergraduate Nursing Degree, Master of Science in Nursing, University of Brescia, Brescia, Italy.

7. Silvia Androni, RN, MSc. Clinical Nurse, Department of Medicine and Surgery, University of Milano-Bicocca, Monza, Italy.

8. Ercole Vellone, RN, MSc, PhD. Assistant Professor in Nursing Science, Department of Biomedicine and Prevention, University of Rome Tor Vergata, Rome, Italy 
9. Barbara Riegel, RN, PhD, FAHA, FAAN. Professor of Nursing, University of Pennsylvania, Philadelphia, Pennsylvania, United States of America.

10. Stefania Di Mauro, RN, MSc. Associate Professor in Nursing Science, Department of Medicine and Surgery, University of Milan-Bicocca, Monza, Italy.

\title{
CORRESPONDING AUTHOR
}

1) Davide Ausili, RN, MSc, PhD. Research Fellow in Nursing Science, Department of Medicine and Surgery, University of Milano-Bicocca, Monza, Italy. Via Cadore 48, 20099, Monza, Italy. davide.ausili@unimib.it. Phone: +390264488032, FAX: +390392332576.

\begin{abstract}
Aims. To describe self-care as defined by the Middle-Range Theory of Self-Care of Chronic Illness and to identify clinical and socio-demographic determinants in a T2DM population. Methods. A multicentre observational cross-sectional study was conducted involving 540 adults with a confirmed diagnosis of T2DM from 6 outpatient diabetes services in Italy. Socio-demographic and clinical data were collected from medical records. The Self-Care of Diabetes Inventory (SCODI) was used to measure self-care maintenance, monitoring, management and confidence dimensions. For each separate scale, scores were standardized 0-100 with higher SCODI scores indicating better self-care; a score $\geq 70$ is adequate. Multiple quantile regression models were performed to identify determinants of each self-care dimension. Results. Self-care maintenance (median $=81.3$ ) and selfcare confidence $($ median $=79.5)$ were adequate in most of the subjects. Self-care monitoring was adequate in only half of the sample $($ median $=70.6)$. Self-care management was poor $($ median $=$ 59.4). Lower self-care maintenance was associated with lower self-care confidence $(\mathrm{p}<0.001)$. Lower self-care monitoring was associated with being male $(\mathrm{p}<0.001)$, having lower self-care confidence $(\mathrm{p}<001)$, and having diabetes for $<10$ years $(\mathrm{p}<0.001)$. Lower self-care management was associated with being male $(\mathrm{p}=0.002)$, being older $(\mathrm{p}=0.005)$, having a low income $(\mathrm{p}=0.030)$,
\end{abstract}


being employed $(\mathrm{p}=0.008)$, having missed diabetes education in the last year $(\mathrm{p}=0.002)$, and lower self-care confidence $(\mathrm{p}<0.0001)$. Lower self-care confidence was associated with having diabetes for $<10$ years $(\mathrm{p}=0.008)$, and having at least one comorbid condition $(\mathrm{p}=0.006)$. Conclusions. Determinants of self-care maintenance, monitoring, management and confidence include both clinical and socio-demographic variables. Modifiable determinants such as self-care confidence and diabetes self-care management education could be used to tailor interventions to improve diabetes self-care.

\section{KEY WORDS}

Diabetes Mellitus, Type 2 Diabetes Mellitus, Self-Management, Chronic Disease, Self-efficacy, Risk factors, Health Education 


\section{INTRODUCTION}

Diabetes is one of the health emergencies of the $21^{\text {st }}$ century affecting 415 million people worldwide and accounting for 5.0 million deaths globally in 2015 [1]. Having diabetes is associated with cardiovascular disease, stroke, diabetic nephropathy, neuropathy, retinopathy, pregnancy complications $[1,2]$, poor quality of life [3, 4], high economic burden [1, 5], and the risk of premature death [1, 6]. Type 2 diabetes mellitus (T2DM) accounts for 90\% of cases of diabetes [7]. Its rise is connected with rising rates of overweight and obesity in adults and youth and a complex interaction of genetic and epigenetic factors [7].

Self-care of T2DM has proven to be cost effective for decreasing complications [8], reducing $\mathrm{Hb} 1 \mathrm{Ac}[9,10]$, reducing hospital admissions $[9,10]$ and therefore costs; it also positively affects quality of life $[9,10]$, promotes empowerment [10] and improves other psychosocial and behavioural factors associated with diabetes $[9,10]$. Unfortunately, little is known about the characteristics of individuals least likely to engage in self-care. Previous studies have shown that T2DM patients with low diabetes-treatment-related quality of life are less likely to perform adequate self-management of insulin injections [11], and that functional status plays a role in patients' health-related quality of life [12]. Knowledge of diabetes following therapeutic education [13] predicts diabetes self-management [14] and quality of life is associated with the quality of diabetes care. However, little information is available on socio-demographic and clinical determinants of self-care in T2DM. Without this knowledge, interventions aimed at promoting selfcare are unable to be targeted to those most likely to benefit.

We examined the socio-demographic and clinical determinants of self-care in adults with T2DM, defining self-care based on the Middle Range Theory of Self-Care of Chronic Illness [15]. This theory has been used widely in the study of cardiovascular diseases [16-18] and other chronic conditions $[9,17,19,20]$. In the theory, self-care is defined as "process of maintaining health through health promoting practices and managing illness" [15]. Self-care comprises three dimensions that interplay: self-care maintenance, monitoring, and management [15] . Self-care 
confidence is not self-care per se, but a factor strongly influencing self-care. Confidence is thought to underlie all self-care behaviours and mediate the relationship between factors such as cognition and self-care behaviours $[16,17,21]$. This theoretical approach acknowledges the dynamic nature and complexity of the self-care process.

According to the theory, self-care maintenance describes those behaviours that improve well-being, preserve health, or maintain physical and emotional stability [15]. In T2DM, these behaviours, either prescribed or self-determined, comprise healthy lifestyle (e.g., appropriate diet, physical exercise, avoiding alcohol and tobacco, foot care), taking medicine as prescribed, and attending medical appointments. Self-care monitoring refers to the monitoring of changes in signs and symptoms such as blood glucose and indicators of hypo/hyper-glycaemia. Self-care management reflects those behaviours used to actively address signs and symptoms when they occur such as adjusting medication or physical activity levels or checking with a health care provider or caregiver. Self-care confidence or self-efficacy is the belief that one has the ability to perform a specific self-care action and to persist in performing that action despite barriers [15]. Self-care by individuals with T2DM has been explored in many studies, [22-25] but the existing literature has a number of important limitations. Determinants of self-care have been described previously but only in relation to single self-care behaviours (i.e. blood glucose monitoring, diet, exercise) [26, 27]. Prior studies have lacked a theoretical framework [28, 29] and therefore self-care of T2DM has not been explored as a dynamic and complex phenomenon with multiple dimensions. Instruments used to measure self-care have not been theoretically based [30, 31]. Comorbid conditions have not been addressed as potentially influencing the self-care process. To address these gaps in the literature, the objectives of this study were: 1) to describe self-care maintenance, self-care monitoring, self-care management and self-care confidence in T2DM patients; and 2) to identify clinical and socio-demographic determinants of self-care maintenance, self-care monitoring, self-care management and self-care confidence in T2DM patients. Having this information can help scientists and professionals to better understand the complexity of self-care in 
T2DM, to identify patients at risk of poor self-care, and to design targeted interventions to improve the health and quality of life of people with T2DM.

\section{METHODS}

A multicentre cross-sectional study was conducted involving T2DM patients from six outpatient diabetes clinics in the North of Italy. Approval was obtained by the Institutional Review Boards of participating centres. Signed informed consent was obtained from all study participants. Study procedures were conducted according to the ethical standards of the responsible committee on human experimentation and with the Helsinki Declaration of 1975, as revised in 2008.

\section{Sample}

We recruited a consecutive sample of 540 adult T2DM patients during outpatient visits. Inclusion criteria were: confirmed diagnosis of T2DM diabetes diagnosed according to guidelines criteria [32] and age $\geq 18$ years. Exclusion criteria were: screening or first visit to the diabetes centre; time since the diagnosis of diabetes of < 1 year; inability to read the study questionnaire; documented cognitive impairment; other types of diabetes (e.g. impaired glucose tolerance or gestational diabetes) [32].

\section{Measurement}

Socio-demographic and clinical data were collected by reviewing medical records. Based on prior studies [20, 24, 29, 33-35], we measured the following potential determinants of self-care: age, gender, education (low = primary or secondary vs. high $=$ higher or academic), income, time since the diagnosis of T2DM as an indicator of experience, presence of comorbidities (at least one vs. none), type of medications (insulin therapy vs. oral blood glucose lowering medications only), self-management education (received vs. not received in the last year). Furthermore, to assess the presence of family support, patients were asked to report if at least one person in their family was giving constant support to them for the management of diabetes (yes vs. not, as self-reported by patients). Standardized criteria from updated clinical guidelines were used to verify the T2DM diagnosis and to assess the presence of comorbidities [32]. Diabetes microvascular complications 
were not included in the count of comorbidities because, according to the theory underlying the study [15], we conceptualized comorbidities as consequences of poor self-care rather than antecedents.

The Self-care of Diabetes Inventory (SCODI), a self-report instrument, was used to measure self-care maintenance, self-care monitoring, self-care management and self-care confidence [36]. The SCODI was developed based on the Middle Range Theory of Self-care of Chronic Illness [15] and demonstrated to be a valid and reliable measure of self-care in the Italian diabetes population [36]. The SCODI had good fit indices and high multiple model based reliability for all four scales (self-care maintenance $=0.81 ;$ self-care monitoring $=0.84$; self-care management $=0.86$; and selfcare confidence $=0.89$ ). Construct validity of the SCODI was demonstrated with an association between SCODI scores and objective clinical criteria such as glycated haemoglobin, body mass index, and the presence of diabetes microvascular complications.

Each of the SCODI scales has dimensions that illustrate different elements of self-care. The self-care maintenance scale has 4 dimensions that measure health promoting behaviours (adherence to diabetes treatments such as diet and medications, reliability $=0.77$ ), health promoting exercise behaviours (adherence to physical activity and active lifestyle, reliability $=0.85$ ), disease prevention behaviours (behaviours aimed at avoiding risk and complications such as vaccination, avoiding alcohol and tobacco, reliability $=0.79$ ), and illness related behaviours (screening examinations, diagnostic tests, and follow-up visits, reliability $=0.95)$ ).

The self-care monitoring scale includes 2 dimensions. The body listening element addresses behaviours like blood glucose or blood pressure monitoring. The symptom recognition element addresses the ability to recognize diabetes symptoms.

The self-care management scale includes autonomous and consultative self-care management behaviour dimensions. Autonomous self-care management involves those behaviours directly performed by patients to manage symptoms or problematic blood glucose levels (reliability 
$=0.89)$. Consultative self-care management involves those behaviours performed in consultation with health professionals or caregivers to solve problematic signs or symptoms (reliability $=0.77$ ).

The self-care confidence scale has 2 dimensions that address task-specific self-care confidence (reliability $=0.90)$, and persistence (reliability $=0.88)$. All the SCODI scales and subscales or dimensions provide a 0-100 standardized score where higher score means better selfcare. A cut-off of 70 points has been used to classify self-care maintenance, self-care monitoring, self-care management and self-care confidence as adequate $(\geq 70)$ or inadequate $(<70)[36]$.

\section{Statistical Analysis}

Socio-demographic and clinical characteristics were described with frequencies and percentages. Box and whiskers plots were used to examine the distributions of the self-care scales. To identify which factor was associated with the self-care scales, median and interquartile range (IQR) were reported for each category and differences tested by means of median test, due to the non-normal distribution of the scales scores. For the same reason, instead of performing linear regression, quantile regression models were adopted to regress the median of scales, and dimension scores, on each variable collected. Thus, the parameter estimated by the model is interpreted as the increment in the median (instead of the mean), for each unit increment in the independent factors. The regression parameters were estimated by the algorithm Simplex due to the number of independent variables included in the models. Confidence intervals and p-values were estimated by using the sparsity function and assuming that errors in the linear model are independent and identically distributed. P-values were considered statistically significant if lower than 0.05.

\section{RESULTS}

The sample of 540 T2DM patients was typically male and most $(77 \%, \mathrm{n}=415)$ were aged 60 years or more. Most were retired $(75 \%$; $=402)$ with a low level (primary or secondary) of education $(83 \% ; n=448)$. A large proportion $(47 \%, n=252)$ had T2DM for 10 years or more. Most $(67 \%, n=363)$ were taking oral glucose lowering medications, but a third $(33 \%, n=177)$ were taking insulin. The vast majority $(87 \%, \mathrm{n}=469)$ had at least one comorbid condition. Only $6 \%$ of the 
sample had received diabetes self-management education in the last year. Socio-demographic and clinical characteristics of the study sample - as well as self-care maintenance, self-care monitoring, self-care management and self-care confidence scores by the same characteristics - are shown in

\section{Table 1.}

Self-care maintenance (median $=81.3, \mathrm{Q} 1-\mathrm{Q} 3:$ 72.9-89.6) and self-care confidence (median $=79.5, \mathrm{Q} 1-\mathrm{Q} 3:$ 65.9-93.2) were adequate in most of the subjects. Self-care monitoring was adequate in only half of the sample (median $=70.6, \mathrm{Q} 1-\mathrm{Q} 3: 55.9-85.3)$. Self-care management was poor $($ median $=59.4$, Q1-Q3: 39.8-75.0). Within self-care maintenance, the health promotion exercise behaviours were the poorest (median $=50.0, \mathrm{Q} 1-\mathrm{Q} 3: 25.0-87.5)$ while illness related behaviours were practiced the most (median $=91.7, \mathrm{Q} 1-\mathrm{Q} 3:$ 75.0-100.0). Body listening was the dimension of self-care monitoring with the highest score (median $=80.0, \mathrm{Q} 1-\mathrm{Q} 3:$ 60.0-90.0) while symptom recognition was borderline (median $=71.4, \mathrm{Q} 1-\mathrm{Q} 3:$ 28.6-92.9). Both autonomous (median $=66.7, \mathrm{Q} 1-\mathrm{Q} 3: 41.7-91.7)$ and consultative (median $=55.0, \mathrm{Q} 1-\mathrm{Q} 3: 35.0-70.0)$ self-care management behaviours were inadequate. In self-care confidence, both task specific (median = 79.2, Q1-Q3: 62.5-95.8) and persistence (median = 80.0, Q1-Q3: 65.0-95.0) self-care confidence were adequate. Self-care maintenance, self-care monitoring, self-care management, self-care confidence and their dimension distributions are reported in Figure 1.

Lower self-care maintenance was associated with lower self-care confidence $(\mathrm{p}<0.001)$. Lower health promoting exercise behaviours were associated with being female $(\mathrm{p}=0.011)$, low income $(\mathrm{p}=0.002)$ and the presence of comorbidities $(\mathrm{p}=0.008)$. Lower disease prevention behaviours were associated with being male $(\mathrm{p}<0.001)$ and being younger than 60 years $(\mathrm{p}=0.001)$. Lower health promoting behaviours were associated with being employed ( $\mathrm{p}=0.039)$, having low education $(\mathrm{p}=0.023)$ and low persistence self-care confidence $(\mathrm{p}<0.001)$. Determinants of self-care maintenance and its dimensions are shown in Table 2.

Lower self-care monitoring was associated with being male $(\mathrm{p}<0.001)$, having lower selfcare confidence $(\mathrm{p}<0.001)$, and having diabetes for $<10$ years $(\mathrm{p}<0.001)$. Lower body listening was 
associated with being male $(\mathrm{p}=0.002)$, and lower task specific self-care confidence $(\mathrm{p}<0.001)$. Lower symptom recognition was associated with being male $(\mathrm{p}=0.006)$, having diabetes for $<10$ years $(p<0.001)$, and lower task specific self-care confidence $(p<0.001)$. Determinants of self-care monitoring and its dimensions are reported in Table 3.

Lower self-care management was associated with being male $(\mathrm{p}=0.002)$, being older $(\mathrm{p}=0.005)$, having a low income $(\mathrm{p}=0.030)$, being employed $(\mathrm{p}=0.008)$, having missed diabetes education in the last year $(\mathrm{p}=0.002)$, and lower self-care confidence $(\mathrm{p}<0.001)$. Lower autonomous self-care management behaviours were associated with being male $(\mathrm{p}=0.043)$, lower task specific self-care confidence $(\mathrm{p}<0.001)$ and higher persistence self-care confidence $(\mathrm{p}=0.0001)$. Lower consultative self-care management behaviours were associated with low income $(\mathrm{p}=0.035)$, having diabetes for $\geq 10$ years $(\mathrm{p}=0.041)$, being employed $(\mathrm{p}=0.019)$, having missed diabetes selfmanagement education in the last year $(\mathrm{p}=0.023)$ and lower task specific self-care confidence $(\mathrm{p}<0.001)$, Determinants of self-care management and its dimensions are reported in Table 4.

Lower self-care confidence was associated with having diabetes $<10$ years $(p=0.008)$ and having at least one comorbid condition $(\mathrm{p}=0.006)$. No statistically significant associations were found between the clinical and socio-demographic variables and the task-specific self-care confidence dimension. Lower persistence self-care confidence was associated with high income $(\mathrm{p}=0.010)$, low education $(\mathrm{p}=0.047)$, and presence of comorbidities $(\mathrm{p}=0.001)$. Determinants of selfcare confidence and its dimensions are reported in Table 5.

\section{DISCUSSION}

The aims of this study were to describe self-care of T2DM patients and to identify the clinical and socio-demographic determinants. We found that self-care maintenance and self-care confidence were adequate in most, self-care monitoring was adequate in half, but self-care management had the poorest score in this T2DM population. Characteristics significantly associated with self-care were confidence, gender, age, income, employment, family support, and length of experience with T2DM, diabetes self-management education, and comorbidity. 
We found that both autonomous and consultative diabetes self-management behaviours were extremely poor in this T2DM population, consistent with our previous results in a sample with concomitant diabetes mellitus and heart failure [20]. It is not surprising that this self-care behaviour is poorest because management requires complex problem-solving and decision making skills as well as the knowledge and skill in managing signs and symptoms [15]. Further, few of these participants received diabetes self-management education in the last year. Persons with T2DM have been shown to benefit from periodic diabetes self-management education [10].

Specific self-care behaviours found to be inadequate or border line were exercise and symptom recognition. Previous investigators have found that physical exercise is suboptimal in people with T2DM $[8,37]$. Exercise behaviours in T2DM are determined by complex physical and psychosocial factors [27] and these factors could represent barriers to exercise behaviours [38]. Symptom recognition was also poor, which is concerning because symptom recognition begins the self-care management process. Others have noted that symptom recognition is affected by the disease (i.e. comorbid conditions or diabetes microvascular complications), and often difficult for people with diabetes $[39,40]$.

Our second aim was to identify clinical and socio-demographic determinants of self-care. We found that lower self-care confidence was associated with all the self-care processes (maintenance, monitoring, and management), as others have found [15, 20, 21, 41]. This is important because self-care confidence can be increased by improving patients' knowledge and skills through therapeutic education [42, 43]. Factors associated with lower self-care confidence included insufficient income, relatively more recent T2DM diagnosis, and having at least one comorbid condition. Others have noted the influence of income on self-care $[35,44]$. Time since diagnosis is a surrogate for experience acquired living with diabetes. Consistent with previous studies, experienced patients may be more confident in their ability to manage a chronic illness [20, 45]. Having a comorbid condition decreases confidence by requiring that the patient consider two or more conditions simultaneously when making self-care decisions [20]. 
Lower self-care monitoring was associated with shorter time since diagnosis. Patients with a more recent diagnosis of T2DM are more likely to perceive themselves as "moderately ill" because they have not experienced severe and long-term disease complications. Thus, consistent with previous studies $[8,20,36,45]$, they seem to postpone persistent self-care monitoring activities.

Poorer self-care management was associated with being male, being older, having a low income, being employed, and having missed diabetes education in the last year. Gender differences in self-care of T2DM patients were found in previous studies $[46,47]$ in which men were shown to be less likely to perform adequate self-care than women. Similar results have been found in other chronic conditions $[20,45]$. However, gender itself - as a determinant of self-care - should be considered with caution because the results differed depending on the specific dimension of selfcare. More evidence exists supporting our finding that older age may be associated with poor selfcare management [48].

Several prior studies have shown that low income is a barrier to T2DM self-care [44, 49]. However, we found that being employed was associated with worse self-care management, consistent with research in other patient populations [50]. It may be that those who are actively working face more competing demands on their time. This conclusion is supported both by previous research [22] and our clinical experience, suggesting that self-care may be less of a priority when work activities are pressing. Finally, lack of recent diabetes education was associated with poor self-care management, confirming the importance of diabetes self-management education (DSME) in improving self-care in T2DM patients [37, 51].

Finally, when we examined the dimensions of each self-care behaviour (i.e. body listening and symptom recognition as dimensions of self-care monitoring) we found evidence that certain variables were protective for one dimension and a risk factor for another one. For example, men were more likely to exercise but less likely to practice illness prevention behaviours. This discrepancy is reflected in other studies that found males to be more physically active but also more likely to smoke and drink alcohol $[52,53]$. 


\section{Strengths and limitations}

To the best of our knowledge, this is the first study investigating the whole self-care process in a T2DM population. This is relevant because the main limitation of previous research was that the complexity of self-care was not acknowledged. Furthermore, this study was the first to use a psychometrically sound, theoretically grounded measure of diabetes self-care, addressing another limitation of previous research. Finally, the use of a theoretical framework that is widely used to study other chronic diseases contributes to further theoretical development $[15,17,19]$.

This study has several limitations. The sample comes from one country and generalization to another requires caution. The cross-sectional nature of the study is another limitation. Psychological variables that can potentially affect self-care - anxiety or depression - were not measured. However, the study was conducted at multiple centres; the sample was large and was enrolled consecutively to avoid selection bias; self-care was measured by a valid and reliable theory-based tool [36], and main clinical and socio-demographic characteristic of the sample are similar to those in the literature [37], suggesting that these results may be useful internationally.

\section{CONCLUSION}

Based on the results of this study, we encourage T2DM health care providers to focus education on the autonomous and the consultative dimensions of self-care management. For selfcare monitoring, symptom recognition should be emphasized. Another area to be emphasized is building self-care confidence. We demonstrated that task-specific and persistence self-care confidence play different roles in the self-care process. As confidence is an indicator of selfefficacy $[15,41]$, persistence despite barriers is key to promoting self-care maintenance behaviours such as diet and medication adherence [16, 27]. Task-specific self-care confidence is important in body listening and symptom recognition because these behaviours require specific knowledge and skill $[15,36]$. These findings could help healthcare providers to stratify and classify patients at risk of poor self-care, tailoring appropriate and effective interventions. For this reason, we provide a synthesis for clinicians showing the significant influences of each socio-demographic and clinical 
variable on the specific dimensions of self-care maintenance, monitoring, management, and confidence (Supplementary).

The determinants of self-care should be systematically assessed to identify patients at risk of poor self-care. As some of these determinants are modifiable factors (i.e., self-care confidence and recent diabetes self-management education), these factors are reasonable to target in order to improve self-care behaviours. Further research is needed to describe how the self-care process and its determinants change over time and the effectiveness of interventions designed to improve selfcare.

\section{Compliance with ethical standards}

\section{Conflict of interest}

The authors declare that they have no conflicts of interest.

\section{Human and animal rights}

All procedures followed were in accordance with the ethical standards of the responsible committee on human experimentation (institutional and national) and with the Helsinki Declaration of 1975, as revised in 2008 .

\section{Informed consent}

Informed consent was obtained from all patients for being included in the study. 


\section{REFERENCES}

1. International Diabetes Federation (2015) IDF Diabetes Atlas 2015 7th edition. Brussels

2. Alberti KG, Zimmet PZ (1998) Definition, diagnosis and classification of diabetes mellitus and its complications. Part 1: diagnosis and classification of diabetes mellitus provisional report of a WHO consultation. Diabet Med J Br Diabet Assoc 15:539-553 . doi: 10.1002/(SICI)10969136(199807)15:7<539::AID-DIA668>3.0.CO;2-S

3. Scollan-Koliopoulos M, Bleich D, Rapp KJ, et al (2013) Health-Related Quality of Life, Disease Severity, and Anticipated Trajectory of Diabetes. Diabetes Educ 39:83-91 . doi: $10.1177 / 0145721712467697$

4. Timar R, Velea PI, Timar B, et al (2016) Factors influencing the quality of life perception in patients with type 2 diabetes mellitus. Patient Prefer Adherence Volume 10:2471-2477 . doi: 10.2147/PPA.S124858

5. Bommer C, Heesemann E, Sagalova V, et al (2017) The global economic burden of diabetes in adults aged 20-79 years: a cost-of-illness study. Lancet Diabetes Endocrinol 5:423-430 . doi: $10.1016 /$ S2213-8587(17)30097-9

6. Morrish NJ, Wang S-L, Stevens LK, et al (2001) Mortality and causes of death in the WHO multinational study of vascular disease in diabetes. Diabetologia 44:S14 . doi:

10.1007/PL00002934

7. Chen L, Magliano DJ, Zimmet PZ (2011) The worldwide epidemiology of type 2 diabetes mellitus - present and future perspectives. Nat Rev Endocrinol 8:228-236 . doi: 10.1038/nrendo.2011.183

8. Ausili D, Bulgheroni M, Ballatore $P$, et al (2017) Self-care, quality of life and clinical outcomes of type 2 diabetes patients: an observational cross-sectional study. Acta Diabetol 54:1001-1008 . doi: 10.1007/s00592-017-1035-5

9. Song M (2010) Diabetes Mellitus and the Importance of Self-care: J Cardiovasc Nurs 25:9398 . doi: 10.1097/JCN.0b013e3181c5a364

10. Powers MA, Bardsley J, Cypress M, et al (2015) Diabetes Self-management Education and Support in Type 2 Diabetes: A Joint Position Statement of the American Diabetes Association, the American Association of Diabetes Educators, and the Academy of Nutrition and Dietetics. Diabetes Care 38:1372-1382 . doi: 10.2337/dc15-0730

11. Mashitani T, Hayashino Y, Okamura S, et al (2015) Diabetes treatment-related quality of life is associated with levels of self-care activities in insulin injection among Japanese patients with type 2 diabetes: Diabetes Distress and Care Registry at Tenri (DDCRT 8). Acta Diabetol 52:639-647 . doi: 10.1007/s00592-015-0725-0

12. Riandini T, Wee HL, Khoo EYH, et al (2018) Functional status mediates the association between peripheral neuropathy and health-related quality of life in individuals with diabetes. Acta Diabetol 55:155-164 . doi: 10.1007/s00592-017-1077-8

13. Rossi MC, Candido R, Ceriello A, et al (2015) Trends over 8 years in quality of diabetes care: 
results of the AMD Annals continuous quality improvement initiative. Acta Diabetol 52:557-571 . doi: 10.1007/s00592-014-0688-6

14. Karbalaeifar R, Kazempour-Ardebili S, Amiri P, et al (2016) Evaluating the effect of knowledge, attitude and practice on self-management in patients with type 2 diabetes. Acta Diabetol 53:1015-1023 . doi: 10.1007/s00592-016-0905-6

15. Riegel B, Jaarsma T, Strömberg A (2012) A middle-range theory of self-care of chronic illness. ANS Adv Nurs Sci 35:194-204 . doi: 10.1097/ANS.0b013e318261b1ba

16. Vellone E, Pancani L, Greco A, et al (2016) Self-care confidence may be more important than cognition to influence self-care behaviors in adults with heart failure: Testing a mediation model. Int J Nurs Stud 60:191-199 . doi: 10.1016/j.ijnurstu.2016.04.016

17. Jaarsma T, Cameron J, Riegel B, Stromberg A (2017) Factors Related to Self-Care in Heart Failure Patients According to the Middle-Range Theory of Self-Care of Chronic Illness: a Literature Update. Curr Heart Fail Rep 14:71-77 . doi: 10.1007/s11897-017-0324-1

18. Riegel B, Dickson VV (2008) A situation-specific theory of heart failure self-care. J Cardiovasc Nurs 23:190-196 . doi: 10.1097/01.JCN.0000305091.35259.85

19. Clari M, Matarese M, Ivziku D, De Marinis MG (2017) Self-Care of People with Chronic Obstructive Pulmonary Disease: A Meta-Synthesis. The Patient. doi: 10.1007/s40271-017-0218-z

20. Ausili D, Rebora P, Di Mauro S, et al (2016) Clinical and socio-demographic determinants of self-care behaviours in patients with heart failure and diabetes mellitus: A multicentre crosssectional study. Int J Nurs Stud 63:18-27 . doi: 10.1016/j.ijnurstu.2016.08.006

21. Vellone E, Fida R, D'Agostino F, et al (2015) Self-care confidence may be the key: A crosssectional study on the association between cognition and self-care behaviors in adults with heart failure. Int J Nurs Stud 52:1705-1713 . doi: 10.1016/j.ijnurstu.2015.06.013

22. Gomersall T, Madill A, Summers LKM (2011) A Metasynthesis of the Self-Management of Type 2 Diabetes. Qual Health Res 21:853-871 . doi: 10.1177/1049732311402096

23. Bohanny W, Wu S-FV, Liu C-Y, et al (2013) Health literacy, self-efficacy, and self-care behaviors in patients with type 2 diabetes mellitus. J Am Assoc Nurse Pract 25:495-502 . doi: 10.1111/1745-7599.12017

24. Coyle ME, Francis K, Chapman Y (2013) Self-management activities in diabetes care: a systematic review. Aust Health Rev 37:513 . doi: 10.1071/AH13060

25. O'Shea MP, Teeling M, Bennett K (2014) Comorbidity, health-related quality of life and selfcare in type 2 diabetes: a cross-sectional study in an outpatient population. Ir J Med Sci 1971 184:623-630 . doi: 10.1007/s11845-014-1190-4

26. Bonner T, Foster M, Spears-Lanoix E (2016) Type 2 diabetes-related foot care knowledge and foot self-care practice interventions in the United States: a systematic review of the literature. Diabet Foot Ankle 7: . doi: 10.3402/dfa.v7.29758

27. Koponen AM, Simonsen N, Suominen S (2017) Determinants of physical activity among 
patients with type 2 diabetes: the role of perceived autonomy support, autonomous motivation and self-care competence. Psychol Health Med 22:332-344 . doi:

10.1080/13548506.2016.1154179

28. Clark ML, Utz SW (2014) Social determinants of type 2 diabetes and health in the United States. World J Diabetes 5:296-304 . doi: 10.4239/wjd.v5.i3.296

29. Hyman I, Shakya Y, Jembere N, et al (2017) Provider- and patient-related determinants of diabetes self-management among recent immigrants: Implications for systemic change. Can Fam Physician 63:e137

30. Lu Y, Xu J, Zhao W, Han H-R (2016) Measuring Self-Care in Persons With Type 2 Diabetes: A Systematic Review. Eval Health Prof 39:131-184 . doi: 10.1177/0163278715588927

31. Caro-Bautista J, Martín-Santos FJ, Morales-Asencio JM (2014) Systematic review of the psychometric properties and theoretical grounding of instruments evaluating self-care in people with type 2 diabetes mellitus. J Adv Nurs 70:1209-1227 . doi: 10.1111/jan.12298

32. American Diabetes Association (2016) Standards of Medical Care in Diabetes. Diabetes Care 39:

33. Karimy M, Araban M, Zareban I, et al (2016) Determinants of adherence to self-care behavior among women with type 2 diabetes: an explanation based on health belief model. Med J Islam Repub Iran 30:368

34. Ouyang C-M, Dwyer JT, Jacques PF, et al (2015) Determinants of dietary self-care behaviours among Taiwanese patients with type 2 diabetes. Asia Pac J Clin Nutr 24:430-437

35. Walker RJ, Gebregziabher M, Martin-Harris B, Egede LE (2014) Independent effects of socioeconomic and psychological social determinants of health on self-care and outcomes in Type 2 diabetes. Gen Hosp Psychiatry 36:662-668 . doi: 10.1016/j.genhosppsych.2014.06.011

36. Ausili D, Barbaranelli C, Rossi E, et al (2017) Development and psychometric testing of a theory-based tool to measure self-care in diabetes patients: the Self-Care of Diabetes Inventory. BMC Endocr Disord 17: . doi: 10.1186/s12902-017-0218-y

37. Nicolucci A, Kovacs Burns K, Holt RIG, et al (2013) Diabetes Attitudes, Wishes and Needs second study (DAWN2 ${ }^{\mathrm{TM}}$ ): cross-national benchmarking of diabetes-related psychosocial outcomes for people with diabetes. Diabet Med J Br Diabet Assoc 30:767-777 . doi: 10.1111/dme.12245

38. Egan AM, Mahmood W a. W, Fenton R, et al (2013) Barriers to exercise in obese patients with type 2 diabetes. QJM Mon J Assoc Physicians 106:635-638 . doi: 10.1093/qjmed/hct075

39. Naegeli AN, Stump TE, Hayes RP (2010) A psychometric evaluation of the Diabetes Symptom Checklist-Revised (DSC-R) cognitive distress, fatigue, hyperglycemia, and hypoglycemia subscales in patients with type 1 and type 2 diabetes. Diabetes Metab Syndr Obes Targets Ther 3:27-30

40. van Puffelen AL, Heijmans MJWM, Rijken M, et al (2015) Illness perceptions and self-care behaviours in the first years of living with type 2 diabetes; does the presence of complications matter? Psychol Health 30:1274-1287 . doi: 10.1080/08870446.2015.1045511 
41. Riegel B, Dickson VV, Faulkner KM (2016) The Situation-Specific Theory of Heart Failure Self-Care: Revised and Updated. J Cardiovasc Nurs 31:226-235 . doi:

$10.1097 / J C N .0000000000000244$

42. Lee Y-J, Shin S-J, Wang R-H, et al (2016) Pathways of empowerment perceptions, health literacy, self-efficacy, and self-care behaviors to glycemic control in patients with type 2 diabetes mellitus. Patient Educ Couns 99:287-294 . doi: 10.1016/j.pec.2015.08.021

43. Reisi M, Mostafavi F, Javadzade H, et al (2016) Impact of Health Literacy, Self-efficacy, and Outcome Expectations on Adherence to Self-care Behaviors in Iranians with Type 2 Diabetes. Oman Med J 31:52-59 . doi: 10.5001/omj.2016.10

44. Yee LM, McGuire JM, Taylor SM, et al (2016) Factors promoting diabetes self-care among low-income, minority pregnant women. J Perinatol Off J Calif Perinat Assoc 36:13-18 . doi: $10.1038 /$ jp. 2015.134

45. Cocchieri A, Riegel B, D'Agostino F, et al (2015) Describing self-care in Italian adults with heart failure and identifying determinants of poor self-care. Eur J Cardiovasc Nurs 14:126-136 . doi: $10.1177 / 1474515113518443$

46. Caruso R, Arrigoni C, Magon A, et al (2017) Health determinants in italian type 2 diabetes Mellitus (T2DM) patients: A critical gender differences analysis. J Res Gend Stud 7:93-108 . doi: $10.22381 / J R G S 7220176$

47. McCollum M, Hansen LB, Lu L, Sullivan PW (2005) Gender differences in diabetes mellitus and effects on self-care activity. Gend Med 2:246-254 . doi: 10.1016/S1550-8579(05)80054-3

48. Weinger K, Beverly EA, Smaldone A (2014) Diabetes Self-Care and the Older Adult. West J Nurs Res 36:1272-1298 . doi: 10.1177/0193945914521696

49. Compeán Ortiz LG, Del Ángel Pérez B, Reséndiz González E, et al (2016) Self-Care Behaviors and Glycemic Control in Low-Income Adults in México With Type 2 Diabetes Mellitus May Have Implications for Patients of Mexican Heritage Living in the United States. Clin Nurs Res 25:120-138 . doi: $10.1177 / 1054773815586542$

50. Dickson VV, McCauley LA, Riegel B (2008) Work-heart balance: the influence of biobehavioral variables on self-care among employees with heart failure. AAOHN J Off J Am Assoc Occup Health Nurses 56:63-73; quiz 74-76

51. Sicuro J, Charrier L, Berchialla P, et al (2014) Self-management Education by Group Care Reduces Cardiovascular Risk in Patients With Type 2 Diabetes: Analysis of the ROMEO Clinical Trial: Figure 1. Diabetes Care 37:e192-e193 . doi: 10.2337/dc14-1054

52. Ahmed AT, Karter AJ, Liu J (2006) Alcohol consumption is inversely associated with adherence to diabetes self-care behaviours. Diabet Med J Br Diabet Assoc 23:795-802 . doi: 10.1111/j.1464-5491.2006.01878.x

53. Nagrebetsky A, Brettell R, Roberts N, Farmer A (2014) Smoking cessation in adults with diabetes: a systematic review and meta-analysis of data from randomised controlled trials. BMJ Open 4:e004107 . doi: 10.1136/bmjopen-2013-004107 
